\title{
LA RELATION CLIENT À L'HEURE DU NUMÉRIQUE : LE CAS DU MARKETING BANCAIRE
}

Philippe Mouillot

\author{
Management Prospective Ed. | « Management \& Avenir »
}

2019/2 Nº 108 | pages 63 à 82

ISSN 1768-5958

Article disponible en ligne à l'adresse :

https://www.cairn.info/revue-management-et-avenir-2019-2-page-63.htm

Distribution électronique Cairn.info pour Management Prospective Ed..

(C) Management Prospective Ed.. Tous droits réservés pour tous pays.

La reproduction ou représentation de cet article, notamment par photocopie, n'est autorisée que dans les limites des conditions générales d'utilisation du site ou, le cas échéant, des conditions générales de la licence souscrite par votre établissement. Toute autre reproduction ou représentation, en tout ou partie, sous quelque forme et de quelque manière que ce soit, est interdite sauf accord préalable et écrit de l'éditeur, en dehors des cas prévus par la législation en vigueur en France. Il est précisé que son stockage dans une base de données est également interdit. 


\title{
La relation client à l'heure du numérique : le cas du marketing bancaire
}

\author{
Philippe MOUILLOT ${ }^{1}$
}

\section{Résumé}

La numérisation de l'activité bancaire et les stratégies multicanales rendent moins attractive la visite en agence, une éducation qui rend les clients moins sensibles aux conseils dispensés par les conseillers. Dans ce contexte, cet article étudie la stratégie d'une enseigne qui développe un marketing à destination de ses personnels afin de recréer, renforcer et finalement faire vivre l'identité de la marque à des fins de ressource stratégique.

\begin{abstract}
The banking service industry's digitalisation, as well as multichannel strategies turn agencies into less and less attractive places for clients who become independent enough to make their daily operations on their own. This article here investigates a bank brand's strategy, which consists in developing a staff-oriented marketing in order to recreate, reinforce and eventually sustain a brand identity for strategic purposes.
\end{abstract}

1. Philippe MOUILLOT: Maitre de Conférences HDR, spécialiste de marketing international, de stratégie et de comportement du consommateur, ses travaux de recherche concernent l'ensemble des saillances et épiphénomènes qu'il est possible de rencontrer en Sciences de Gestion, notamment en termes de différences culturelles, de comportements institutionnels et de stratégies organisationnelles atypiques. Son axe de recherche majeur concerne la détection et l'accompagnement des HQI (Hauts Quotients Intellectuels) pmouillot@poitiers.iae-france.fr 


\section{Une numérisation galopante}

Cette recherche se penche sur la notion de fidélisation à la marque bancaire par l'étude d'une enseigne qui a choisi de remettre le conseiller au centre de sa stratégie de communication dans un secteur dans lequel le numérique éduque de plus en plus les consommateurs à se passer de la relation humaine. Ce dernier constat n'est d'ailleurs pas un épiphénomène. En France en 2018, grâce à Internet, il est plus facile de comparer les services qui sont offerts d'une banque à l'autre bien que la notion même de «banque » soit devenue complexe à appréhender. En effet, aux banques françaises présentes dans l'hexagone dont le service était composé d'agences et de distributeurs automatiques, soit sept enseignes (i.e. Groupe BNP Paribas ; Groupe Crédit Agricole avec CASA et LCL ; Société Générale ; Groupe BPCE avec Banque Populaire, Caisse d'Épargne et Natixis ; Groupe CM11-CIC avec les 11 fédérations du Crédit Mutuel, le Crédit Industriel et Commercial et ses filiales ; la Banque Postale ; et le Crédit Mutuel ARKEA), s'ajoutent dorénavant des «pure players », ou banques uniquement en ligne, dont le nombre et la performance ne cessent d'augmenter. Pour illustrer cette réalité, citons le palmarès 2018 réalisé par CultureBanque pour la France: ING Direct, une banque en ligne qui regroupe plus d'un million de clients tout en restant très discrète sur ses résultats; Boursorama Banque, la pure player de la Société Générale qui vient d'atteindre le million de clients et qui menace la première place d'ING Direct; Fortuneo Banque, la pure player du Crédit Mutuel ARKEA qui accueille 365000 clients ; Monabank, la pure player du Groupe CM11-CIC avec 310000 clients ; Hellobank!, une structure dédiée aux 300000 clients de BNP Paribas qui ont souhaité basculer à une relation uniquement en ligne ; et BforBank, spécialiste de l'épargne en ligne qui propose un compte courant à ses 165000 clients. Cette photographie est évidemment éphémère tant l'offre bancaire fluctue au grès du développement des nouvelles technologies et surtout des comportements des utilisateurs envers les solutions numériques : les vieux réflexes concurrentiels du passé sont restés les mêmes ; ils ont simplement changé de garde-robe.

L'actualité du marché de la banque de détail, c'est-à-dire à destination des épargnants et des clients non-professionnels de la finance, est donc intimement imbriquée à celle des nouvelles technologies. Car force est de constater que grâce à Internet et aux différentes applications proposées comme outils numériques de leur quotidien, les clients d'enseignes bancaires ont non seulement développé leur connaissance de l'environnement financier qui les concerne, mais surtout leur indépendance même si de nombreuses résistances persistent encore (Notebaert et Attuel-Mendes, 2010). Les clients des banques sont aujourd'hui capables de comparer rapidement des offres autrefois jugées complexes, et la loi Macron de mobilité bancaire vient renforcer la loi Hamon et les efforts entamés par les pouvoirs publics depuis 2004 pour forcer les banques à faciliter le changement de domiciliation pour les particuliers, le tout gratuitement. Le client est donc (re)devenu tout puissant car il est aujourd'hui capable de mettre la clôture de tous ses comptes dans la balance d'une négociation, de 


\section{La relation client à l'heure du numérique : le cas du marketing bancaire}

comprendre sans failles les compositions de profils investisseurs, et surtout de diversifier seul son portefeuille d'outils en sollicitant, par exemple, une banque pour ses placements et un pure player pour ses outils de paiement. Et les jeunes ne sont pas en reste; autrefois chasse gardée de leurs parents, ils sont maintenant capables très tôt de gérer leur argent, simplement grâce à leur virtuosité d'assimilation de solutions numériques.

Cette frénésie de développement technologique ne va pas sans rappeler la théorie du point d'inflexion d'Andrew Grove (1998), point à partir duquel tous les fondamentaux d'un marché ou d'une activité sont sur le point de changer drastiquement et surtout irrémédiablement, la plupart du temps sous l'influence de l'apparition de nouveaux comportements ou de nouvelles technologies.

En 2017, l'étude SAB/CGI a mis en exergue que les cinq nouveaux services bancaires incontournables en 2018 sont respectivement (1) la banque mobile (i.e. gestion des opérations courantes via son smartphone), (2) les services d'aide à la gestion des comptes (i.e. analyse en ligne de gestion de ses dépenses afin de mieux utiliser son argent), (3) les nouveaux moyens de paiement (i.e. paiement sans contact), (4) les chatbots (i.e. messageries instantanées avec des robots de conversation), et (5) les cartes bancaires « intelligentes » (i.e. Revolut, Max, Sharepay, des outils permettant d'agréger plusieurs cartes, d'effectuer des paiements multidevises, de mettre en place un partage automatique des dépenses, etc.).

Une question se pose alors inexorablement: qu'en-est-il de l'intérêt et de l'utilité de l'agence bancaire ? En effet, la banque doit-elle se réinventer en termes d'expérience client humaine ou bien laisser le numérique totalement dominer le marché ? Il y a là une vraie question stratégique car non seulement la bulle numérique peut atteindre des limites de tolérance technique comme psychologique pour le client, notamment par le degré d'intrusion des enseignes dans la vie privée, mais en plus, la relation humaine entre le conseiller et le client peut totalement être repensée, ouvrant ainsi la voie à de nouvelles natures d'avantages concurrentiels durables. En effet, si les clients ont tendance à rapidement adouber les possibilités techniques qui leur simplifient la vie, l'argent reste l'argent, un objet avec lequel on ne voit jamais d'un très bon œil le transfert de contrôle auprès de machines et autres algorithmes. D'ailleurs, la notion d'ingérence est de plus en plus prégnante dans le secteur bancaire puisque l'optimisation des activités de gestion financière passe nécessairement par une analyse détaillée de ces dernières, ce qui donne parfois lieu à des actions créant plus de dissonance cognitive que d'avantage comparatif dans l'esprit des consommateurs. La question de la place du conseiller bancaire dans l'environnement numérique est donc centrale au point que certaines enseignes choisissent de prendre le contrepied de la numérisation à outrance pour revenir à une construction de la fidélisation fondée sur l'humain plutôt que sur l'instrument. Mais dans ce cas, comment mobiliser son personnel pour qu'il réussisse à se projeter dans une stratégie dans laquelle son importance, donc sa responsabilité, serait à nouveau source d'avantage concurrentiel ? C'est cette 
réflexion qui a fait naître notre question de recherche : face à la performance théoriquement supérieure de la machine, un marketing à destination des salariés d'une banque peut-il replacer le conseiller au centre de l'échiquier en tant qu'acteur porteur de l'empathie et du discernement nécessaires au renforcement de la fidélisation de la clientèle ? Plus simplement, une communication interne peut-elle réhabiliter le conseiller bancaire dans son positionnement originel de créateur de valeur, donc d'avantage concurrentiel, face à la technologie ?

Nous proposons d'étudier cette question avec le cas d'une enseigne bancaire qui a souhaité conserver l'anonymat, et par l'ancrage théorique du prisme identitaire (Kapferer, 1988) et de l'échange social (Homans, 1958). En effet, lors de nos entretiens, nous avons obtenu confirmation que cette enseigne avait choisi de remettre le conseiller au cœur de la création de valeur de la banque dans l'esprit du consommateur à l'aide d'une stratégie de communication élaborée à destination de ses employés sans pour autant les en informer. Ceci n'allait pas sans rappeler les travaux de George Homans (1958), auteur de la théorie de l'échange social qui met en avant l'idée selon laquelle toutes les relations se forment, se maintiennent ou se brisent en raison d'une analyse coûts - bénéfices nous amenant à réaliser des comparaisons entre les alternatives proposées et à choisir les relations qui nous procurent donc le plus de bénéfices à moindre coût.

Quant au prisme identitaire de Jean-Noël Kapferer (1988), les représentations et les liens sociaux étant utiles à la création d'une relation commerciale durable, l'identité de marque devient souvent naturellement le fer de lance d'une stratégie de communication. Elle est son socle, les valeurs qui lui permettent d'être ce qu'elle est et ce qu'elle représente. Lorsque l'image est fluctuante et le positionnement modifiable, soit par décision stratégique, soit sous la pression des consommateurs, l'identité est peu sensible aux influences extérieures puisqu'on ne peut effacer un nom ou une histoire. L'identité de la marque est donc la partie la plus importante de la base communicationnelle d'une entreprise car elle est à la fois connue, stable et inaltérable. Pour en cerner toute la symbolique, le prisme développé par Kapferer en 1988 regroupe les variables de physique, de personnalité, de culture, de relation, de reflet et de mentalisation de la marque permettant de la contextualiser en termes d'inconscient collectif et d'existence d'un lien social, un outil qui nous permet ici de suggérer à cette enseigne des pistes d'évolution.

L'association de la théorie de l'échange social et du prisme identitaire offre ici une perspective intégrative en ce sens que la création de valeur participe d'une démarche plus complexe que le simple cadre pécuniaire. En effet, si la banque est une réelle forme de représentation sociale de la gestion par le contrôle rationnel, elle n'en demeure pas moins également une organisation susceptible de créer de la valeur par d'autres instruments et d'autres vecteurs intangibles. Aussi, considérer la création de valeur grâce à un échange social interne à l'organisation mérite d'étendre l'impact d'une telle stratégie à l'image de marque de l'enseigne. Plus précisément, en identifiant ses composantes les 


\section{La relation client à l'heure du numérique : le cas du marketing bancaire}

plus sensibles à toute évolution de son positionnement, la marque vérifie la cohérence de son mix, détermine d'éventuels nouveaux leviers marketing et limite toute forme de dissonance cognitive dans l'esprit du prospect. Dans ce contexte méthodologique, à l'issue d'une revue de la littérature en lien avec la fidélisation, la marque et les stratégies multicanales, principalement dans l'environnement du marketing bancaire, nous présenterons notre terrain, puis nous justifierons nos outils d'analyse et proposerons une discussion suggérant des avancées managériales avant de conclure sur les limites de cette étude.

\section{Banque et image de marque}

De la même manière qu'il existe plusieurs entreprises sur un même secteur, voire segment, il existe plusieurs banques proposant les mêmes services, ce qui aboutit à une concurrence qui force ses acteurs à considérer des notions récurrentes à chaque activité commerciale, notamment celles de fidélisation du client (Salerno, 2001 et 2002), de taux de pénétration de la marque, de positionnement ou encore d'avantage comparatif. Mais le marché bancaire ne cesse d'évoluer. En effet, non seulement des enseignes internationales se développent avec des offres locales mais, en plus, le métier lui-même est en pleine mutation, notamment par la digitalisation de l'activité bancaire ou encore l'apparition d'activités nouvelles de type financement participatif ou encore agences de courtage (Strauss, 2013). Cette évolution a également abouti à une transformation comportementale des clients. Car s'il fut un temps où l'on hésitait longtemps avant de changer de fournisseur de services bancaires, ce n'est aujourd'hui plus le cas, d'une part grâce à la facilité que procurent les démarches électroniques et, d'autre part, grâce à la multiplication des solutions alternatives, nous l'avons vu. Dans ce contexte, l'orientation marque est devenue prépondérante dans le secteur bancaire, à la fois du point de vue des managers et des employés. À ce titre, Elaine Wallace et al. (2013) montrent à la fois comment les comportements des employés créent (Ambler et Barrow, 1996; Balmer, 1995), renforcent (Miles et Mangold, 2004) et font vivre l'identité de la marque au point d'en faire une réelle ressource stratégique. Ils montrent également comment cette marque sert de balise, inspirant les employés et accroissant leur adoption des valeurs associées à la marque (Gromark et Melin, 2011).

En parallèle de cette évolution comportementale des clients, la banque doit gérer leur nature. Car l'activité bancaire est celle de tout le monde, surtout des jeunes consommateurs (18-25 ans) qui ont besoin de financer leurs études et de s'installer dans la vie (Glémain, 2014) : les analystes financiers évaluent à environ 400000 le nombre de «jeunes » qui arrivent ainsi sur le marché de l'emploi sans être équipés d'outils de gestion bancaire. Les cibles sont donc multiples et diversifiées (Rico et Sueur, 2014 ; Valette-Florence et de Barnier, 2013), ce qui complexifie la démarche marketing. Par exemple, les jeunes utilisateurs de services bancaires accordent plus d'importance à la relation au service que les adultes qui, quant à eux, privilégient la relation au conseiller (Mercanti-Guérin, 2011). Les nouvelles technologies contribuent d'ailleurs 
fortement à fragiliser cette forme de relation puisque bon nombre de services en ligne se sont maintenant substitués à l'action du conseiller (Tournois, 2017). Si les stratégies de distribution multicanales s'imposent désormais grâce à l'offre numérique comme l'un des modèles de gestion les plus actifs (Vanheems, 2009), la banque n'est pas en reste face à cette évolution. En effet, si les clients utilisateurs des services bancaires deviennent «mixtes» en faisant appel à différents canaux pour réaliser leurs opérations, ils doivent faire l'objet d'une attention particulière puisque non seulement ils montrent leur propension à l'indépendance mais en plus leur relation au conseiller devenant rare, elle devient du même coup précieuse.

La banque est donc dorénavant contrainte d'être toujours plus compétitive et comparative, non plus seulement en termes de performance financière ou d'image mais également en termes de relation client (Vanheems, 2013), surtout à l'heure des stratégies cross canal et omni canal où la dimension sociale revêt toujours plus d'importance (Collin-Lachaud et Vanheems, 2015). La complexité de ses offres (Carlsson Hauff et al., 2014) exige une relation humaine minimale, ce que l'évolution comportementale et le partage de compétences ont tendance à réduire. Lentement mais sûrement, la banque perd donc de son humanité pour un rôle mécanique de gestion financière ou d'agrégation d'outils utilisateurs, laissant ainsi peu de place aux salariés en termes de création de valeur. Face à cette mutation, la banque doit mettre en place des stratégies afin de revenir à ses valeurs (Nyman, 2014), ses politiques, et surtout ses personnels, garants de certains liens sociaux issus de représentations de même nature.

Ainsi, pour Nathalie Gardes et ses collègues (2013), les représentations sociales des individus à l'égard des marques bancaires reposent sur les dimensions d'attribut, d'univers évoqués et d'entreprise ; autrement dit, les clients d'une banque se la représenteraient au travers de ses produits et services spécifiques, de ses promesses (i.e. accueil, performance) et de son poids ou de sa notoriété dans l'espace économique ad hoc plutôt qu'au travers de ses salariés.

La question de la nature de la relation à la marque bancaire n'est d'ailleurs pas nouvelle (Heinonen, 2014). Depuis une dizaine d'années, les chercheurs questionnent notamment le rôle de l'employé bancaire dans la création de valeur de marque au point de considérer le potentiel communiquant des agents lors de leur recrutement. Ces chercheurs (Knox et Freeman, 2010 ; Papasolomou et Vrontis, 2006) défendent notamment l'idée que les employés des agences bancaires peuvent parfois être considérés comme des clients internes dont la performance commerciale est liée à la qualité de la formation qu'ils reçoivent de la part de l'institution après qu'ils ont été recrutés, ainsi qu'à la valeur de leurs primes. Depuis, l'idée d'un client interne nourrit donc volontiers la tentation d'explorer de nouvelles solutions pour développer la fidélité à la marque bancaire. Et là encore, on observe que confiance et fidélité résultent de la durée et de la qualité de la relation interpersonnelle existant entre le client et le personnel de la banque (Gatfaoui, 2007). Ce paradigme est renforcé par l'idée que, dans le secteur bancaire, la confiance et le degré d'engagement dans la relation 


\section{La relation client à l'heure du numérique : le cas du marketing bancaire}

professionnelle gèrent la médiation de la totalité de l'impact de la satisfaction du client, notamment en termes de prospective (Boke Chakiso, 2015).

La transaction commerciale bancaire est alors parfois perçue comme résultant d'un lien social, un lieu de rencontre, notamment avec les travaux de Fulvio Fortezza et Tonino Pencarelli (2011) qui remettent l'employé au centre de la création de valeur expérientielle, ou encore d'Anne Julien (2013) qui évoque l'importance pour les banques de créer des réseaux sociaux nonvirtuels, capables de créer une solidarité concrète entre de nombreuses parties prenantes. En complément, Gilles N'Goala (2010) soutient que la confiance du client augmente à mesure que l'engagement affectif devient prépondérant dans l'échange relationnel, ce qui nous ramène à la théorie de l'échange social de George Homans (1958).

La littérature met donc en exergue une série de réalités qui rendent la composition de l'univers bancaire complexe, voire paradoxale puisque le client recherche à la fois la distance par des instruments de gestion efficaces et la proximité par une relation humaine rassurante. Ce paradoxe est renforcé par la présence de consommateurs de plus en plus «mixtes» (Vanheems, 2009) puisqu'ils personnalisent désormais leur consommation et leurs outils de gestion grâce à la disponibilité de différents canaux d'information et d'action. Face à cette recrudescence technologique de pure players ou de canaux qui en sont inspirés au sein des banques traditionnelles, le salarié est naturellement réhabilité. Mais il fait alors face à un degré d'exigence supérieur au passé puisqu'il n'est désormais plus considéré par le client comme un élément habituel de la structure bancaire mais bien comme un créateur unique de valeur également unique dans l'acception de la théorie de l'échange social (Homans, 1958).

Pour résumer l'actualité sociétale du secteur bancaire français, nous pouvons donc dire que nous sommes aujourd'hui face à une banque devenue multicanale, à destination de clients multi-profils, le tout dans une réalité intergénérationnelle dans laquelle le numérique distancie la relation des jeunes au conseiller, fragilise la perception des seniors car ils sont moins connectés, augmente l'atomicité du marché et déclenche des guerres de prix, ce qui menace jusqu'à l'avenir même du concept d'agence bancaire. Dans ce maelstrom concurrentiel, promesses et territoires de marques bancaires sont dorénavant dilués au point que le client utilise une organisation bancaire séquencée, un peu à l'instar d'un gourmet qui choisirait l'entrée exotique d'un restaurant, le plat peu calorique d'un autre et un dessert livré à domicile. La satisfaction du client devient donc difficile à mesurer puisque la marque en tant que porteur de solutions globales disparaît au profit d'une mosaïque de services dématérialisés pour lesquels le risque de dissonance cognitive augmente et l'étude de la satisfaction doit se faire soit en termes de satisfaction globale, soit en termes de satisfaction cumulée, soit en termes de satisfaction liée à chaque épisode de la démarche bancaire. De plus, l'absence de lieu de rencontre, donc de création de lien social et affectif concret permettant la construction d'une relation bienveillante, n'aide pas à la construction d'une relation de fidélisation 
entre les différents acteurs. Nous sommes donc bien dans une situation de recherche où le recours au double ancrage théorique Homans-Kapferer prend tout son sens. En effet, l'avènement des stratégies cross canal et omni canal ont pour principale conséquence de faire revêtir toujours plus d'importance à la dimension sociale. La confiance et la fidélité résultent principalement de la durée et de la qualité de la relation interpersonnelle existant entre le client et le personnel de la banque. Également, la transaction commerciale bancaire est parfois perçue comme résultant d'un lien social. Par conséquent, stratégies multicanales, confiance et fidélité, et transaction commerciale bancaire nourrissent ensemble une approche par la théorie de l'échange social. Quant au prisme identitaire de Kapferer, les représentations sociales des individus à l'égard des marques bancaires reposant sur des dimensions qui excluent les salariés, il apparaît justifié de tenter de modifier ces représentations par une modification du prisme identitaire de la marque bancaire.

Cette synthèse est très importante dans le cas qui nous concerne puisque, justement, on y trouve la justification de notre recherche. En effet, notre étude de cas nous a permis d'effectuer une observation participante, notamment auprès de plusieurs acteurs d'enseignes bancaires.

Les données collectées ont permis de montrer qu'il existe au moins une enseigne qui a choisi une stratégie de communication marketing innovante pour remettre le conseiller au cœur de la création de valeur bancaire.

\section{Méthodologie et collecte des données}

Notre méthodologie a consisté en la mise en œuvre d'une observation participante périphérique afin de pouvoir procéder à des entretiens semi-directifs. La notion d'observation participante est souvent mobilisée lors de réalisation d'enquêtes de terrain. Egalement connue dans son acception de recherche en Sciences de Gestion par l'étude de cas, elle associe une grande pertinence grâce à une discussion sur le rôle de l'acteur tantôt participant qui observe, tantôt observateur qui participe (Soulé, 2007). En outre, bien que cette approche ait du mal à s'insérer dans un cadre conceptuel précis puisqu'elle est alternativement considérée comme méthode, objet d'étude exploratoire, recherche-action ou encore technique qualitative (Hlady-Rispal, 2015), elle autorise observation et questionnement sur une distorsion inhabituelle dans le cadre de processus fortement normés. En ce qui concerne notre étude de cas, nous avons eu un rapport au terrain qui nous a permis de mettre en place une observation participante périphérique, c'est-à-dire que nous nous sommes cantonnés à n'étudier qu'un aspect marketing de la communication de l'enseigne, et ce auprès d'un panel réduit de répondants. En outre, nous avons souhaité que ces derniers appréhendent notre travail comme une étude de nature journalistique plutôt que scientifique afin d'augmenter leur liberté de parole. Cette observation a permis de retrouver dans les réponses des salariés une relation marqueemployés qui est un antécédent majeur de l'identification organisationnelle 


\section{La relation client à l'heure du numérique : le cas du marketing bancaire}

et de comportements congruents, soit envers la marque, soit orientés clients (Löhndorf et Diamantopoulos, 2014). Faisant partie des trois banques en ligne qui regroupent des volumes comparables de clients (i.e. Fortuneo Banque, pure player du Crédit Mutuel ARKEA, 365000 clients; Monabank, pure player du Groupe CM11-CIC, 310000 clients ; et Hellobank !, pure player de BNP Paribas, 300000 clients), justifiant ainsi de fortes remises en question concurrentielles, nous avons choisi cette enseigne car la marque montrait des signaux faibles de retour à la tradition du conseiller par ses supports de communication rencontrés par sérendipité. Fin 2016, début 2017, dates de nos observations participantes, l'enseigne gère 7 milliards d'encours de crédit et 10 milliards d'épargne. Elle offre tous les types de produits bancaires, des produits d'assurance, et un service d'expertise immobilière dans une zone de chalandise comprenant plusieurs départements. Son autonomie dans le domaine de la communication s'explique par sa structure décentralisée. Celle-ci comprend trois niveaux. Le premier est constitué par les caisses locales, financièrement autonomes, qui réalisent les opérations de banque et de distribution des produits d'assurance, et qui gèrent en tout 10,4 millions de clients et 6,5 millions de sociétaires. Le deuxième correspond aux groupes régionaux qui sont l'organe de stratégie, de contrôle et de représentation locale de la marque. Le dernier est celui d'une confédération qui assure le contrôle des groupes régionaux. Elle a notamment en charge la représentation auprès des pouvoirs publics et la gestion d'une caisse centrale.

\subsection{Un marketing innovant?}

Au moment de notre observation, la communication marketing de l'enseigne était constituée des traditionnels supports que l'on retrouve habituellement en agence, à l'instar de certains pure players qui, confrontés aux limites d'une présence exclusivement virtuelle, ont implanté des points de vente ou des agences, par exemple Amazon. Le réseau au sein duquel chaque canal avait pour mission de desservir un segment particulier de clientèle s'est ainsi progressivement imposé comme modèle de gestion. Pourtant, ce multicanal (chaque canal s'adresse à un segment spécifique de clientèle) semble aujourd'hui laisser la place au transcanal (plusieurs canaux sont offerts à un même client pour des occasions d'achat différentes), voire au crosscanal (plusieurs canaux sont proposés à un même client pour une seule occasion d'achat; Belvaux et Notebaert, 2018). Cependant, à l'heure d'une attraction de plus en plus marquée vers les pure players grâce au rajeunissement des utilisateurs et à la simplification des outils de gestion à distance, le fond des messages de la marque étudiée ne mettait pas spécifiquement en exergue des items de type numérique comme la plupart de ses concurrents. En effet, la communication de cette enseigne était alors axée sur le devoir de conseil du personnel d'agence. Nul doute que cette banque ne sous-entendait alors pas d'échanger avec des chatbots mais invitait bien à la création d'une relation sociale et du développement d'un lien, sinon affectif, tout au moins récurrent avec «son » conseiller. 
Mais là où la subtilité marketing de l'enseigne étudiée se dessine de manière plus précise, c'est dans la composition même de ses messages. En effet, à la lecture des différents prospectus à disposition des clients, on constate que le message engage fortement la responsabilité des employés des agences quant à la création, l'instauration et le développement d'une relation humaine de confiance à la fois stable et durable. Ici, la marque a choisi d'orienter sa communication vers ses propres conseillers comme variable proactive et non plus réactive de création de valeur. Ainsi, des slogans locaux proposant les photos des employés, les noms des responsables, ou encore des solutions locales « à l'ancienne » comme, par exemple de mettre de la monnaie à disposition des professionnels les jours de marché, ont été fabriqués afin de responsabiliser le personnel des agences bancaires à la mission de création de lien, prenant ainsi le contrepied en amont d'autres enseignes de la tendance à la dématérialisation de la relation bancaire type HSBC.

Le diagnostic de la pertinence de cette communication du siège vers les clients et du siège vers les employés, communication mise en place à la fois pour informer les clients et pour sensibiliser l'employé sur son rôle éminemment important de créateur de lien social, que ce soit face à la gestion quotidienne des comptes dont il a la responsabilité ou à l'élaboration d'un projet, a donc été la raison d'être de cette étude. Pour répondre à la fois à la question de la prise de conscience des responsables d'agence de la stratégie marketing issue du siège de notre enseigne et à celle de l'impact du lien social et affectif de cette stratégie sur la relation commerciale, des informations furent recueillies au mois de septembre 2016 au sein du siège régional de la banque à l'aide d'entretiens semi-directifs d'une durée de 90 minutes conduits auprès de sept responsables d'agences régionaux, ainsi qu'au mois de janvier 2017 conduits auprès des panels suivants : profils clients épargnants de la banque étudiée (5 sujets), profils clients investisseurs de la marque étudiée ( 5 sujets), clients d'autres établissements bancaires (10 sujets), et salariés d'agences de l'enseigne étudiée (10 sujets). Les thématiques des entretiens effectués auprès des responsables d'agence régionaux portaient sur (1) leur connaissance du prisme identitaire de leur marque, (2) la nature de l'engagement de leurs salariés étant en contact avec la clientèle et (3) la typologie du taux de rotation de leur personnel. Les thématiques des entretiens effectués auprès des autres panels portaient sur (1) les raisons de leur fidélité ou de leur déception, (2) les sources perçues de création de valeur et (3) leur perception de la notion de «banque » face aux pure players et aux offres multicanales.

Nous avons choisi d'interroger des chefs d'agence afin de mesurer leur degré de connaissance et d'implication dans ce processus marketing, ainsi que des employés afin de déterminer si la valeur créée à partir de la relation client était issue d'une action naturelle des employés ou bien conséquente à la mise en œuvre d'une stratégie marketing visant à les stimuler. Afin d'en pondérer les résultats, nous avons souhaité compléter nos données par une série d'entretiens avec des épargnants, clients de la marque étudiée, en postulant que ces derniers devraient sans doute être plus sensibles à la relation client puisque 


\section{La relation client à l'heure du numérique : le cas du marketing bancaire}

peu connaisseurs des produits financiers, des clients investisseurs, sans doute plus indépendants dans leur gestion (Julien, 2013 ; Renault, 2017), et d'autres clients afin de vérifier que cette banque se distinguait vraiment d'autres enseignes au travers de son altérité.

Les données ont été recueillies dans le cadre d'un protocole de confidentialité rigoureux. Un responsable d'agence (depuis ayant quitté l'enseigne) avait organisé une rencontre avec deux autres responsables dans ses bureaux. Cependant, salariés comme employés ont été interrogés hors de leurs cadres professionnels afin de limiter les biais cognitifs. Tous ont refusé que les entretiens soient enregistrés mais les prises de notes furent possibles. En matière de traitement des données, le recours aux verbatim a été préféré aux méthodes de type Alceste ou NVivo afin de privilégier la détection de sentiments et de ressentis face à des analyses plus lexicologiques et lexicométriques. Les verbatim sélectionnés l'ont été sur la base de leur propension à clairement illustrer les opinions majoritaires.

\subsection{La position des responsables d'agences}

L'intérêt d'échanger avec les responsables d'agences était de mesurer leur degré de connaissance et d'implication dans le processus marketing ici étudié. Les données recueillies ont indiqué que la communication était imposée par le siège et que les directions locales ne se posaient généralement pas de questions quant à sa pertinence : (i.e. verbatim : «Oh vous savez, nous recevons packages et instructions »; «nous sommes des financiers, pas des marketeurs »; « difficile d'aller à contre-courant des instructions issues des instances nationales »). Convaincues que les outils marketing sont uniquement destinés aux clients et aux prospects (i.e. verbatim : «Les brochures sont pour nos clients»; «la publicité c'est pour les clients, évidemment»; « pourquoi me demandez-vous ça, n'est-ce pas évident?»), les directions locales ont eu des réponses formatées quant aux valeurs de la marque (i.e. verbatim : «Une force de vente totalement dédiée à l'information du client»; « une banque avec laquelle il est possible de communiquer »; " priorité aux jeunes! 》) et aux indicateurs de performance de leur personnel. Pour cela, elles se fondent sur les ratios habituels de rotation de personnel, de rendez-vous transformés en signatures de contrats ou d'ouvertures de comptes, de taux d'absentéisme et de nature de produits vendus (e.g. assurances-vie vs. Livrets A) (i.e. verbatim : «Nous avons des incentives précis constitués de primes et de moments conviviaux où l'on félicite autant une action remarquable que la naissance d'un enfant»; «oh comme partout vous savez, degré de mobilité, ancienneté, ouvertures de comptes, absentéisme, etc. »; «ce qui motive les gens cher monsieur, c'est l'argent; cela est et a toujours été! "). La possibilité d'une stratégie marketing à destination du «client interne » leur a totalement échappé (i.e. verbatim : «Que voulez-vous dire?»; «je ne comprends pas votre question ») au point de ne pouvoir émettre que des spéculations au sujet de leurs personnels (i.e. verbatim : «J'imagine qu'ils sont heureux de nous voir si actifs»; « "je pense qu'ils n'ont pas de problèmes sinon ils m'en parleraient»), 
notamment en termes de gestion des démissions (i.e. verbatim : «Elles sont gérées par l'antenne régionale»).

À la lecture de nos informations, les responsables d'agence ne semblaient donc ni concernés ni engagés dans la mise en place d'une stratégie de communication susceptible de soutenir leurs actions managériales habituelles de motivation du personnel pour accueillir et fidéliser les clients visiteurs de l'agence ou les prospects. Avec une faible sensibilité aux outils marketing de l'enseigne de la part des responsables régionaux et une méconnaissance des leviers marketing inhérents à la stratégie de la marque, une réflexion au sujet de son prisme identitaire semble donc nécessaire.

\subsection{La perception des salariés}

L'objectif des échanges avec le personnel était de s'adresser à la cible directe de la stratégie de communication de notre marque bien que cela ne soit en rien exposé par la direction. Nous souhaitions ici déterminer l'origine naturelle ou artificielle de l'action commerciale des employés envers leurs clients dans le cadre d'une acception du fait que la relation humaine était source de création de valeur. Les résultats ont montré que les répondants étaient principalement fidèles à l'enseigne par adhésion à sa philosophie (i.e. verbatim : «Je suis heureuse d'avoir un travail, d'autant plus dans une banque qui me ressemble »; "je me sens bien ici, à ma place, au milieu de valeurs qui me sont chères "; "j'aime accompagner de nouveaux projets, surtout auprès des jeunes, et cette banque m'en donne les moyens »), ce qui les plaçaient systématiquement en position de transmettre cette adhésion au prospect (i.e. verbatim : «Connaissez-vous bien nos produits? ; « nous développons une réelle relation humaine» ; «avez-vous des enfants?»). Motivés par les encouragements reçus de la part de leur hiérarchie (i.e. verbatim : «on me propose régulièrement des formations internes» ; « les primes sont très attractives »; "j'ai facilement accès à mon directeur pour échanger »), les répondants furent ici relativement unanimes quant à la place des chiffres face à l'humain (i.e. verbatim : «Ils sont la base de beaucoup de choses mais rien ne remplace la relation humaine»; "je crois en eux, ce qui n'est pas toujours le cas de la part de nos clients, d'où ma contribution et, dans une certaine mesure, ma valeur ajoutée » " une banque sans chiffres c'est surtout une banque sans clients »). Mais c'est lorsque l'on a abordé la critique du message marketing de l'enseigne que les réponses des employés interrogés ont été les plus troublantes. En effet, la mesure de leur fidélité à l'enseigne a été très révélatrice du rôle de la stratégie de communication de la marque (i.e. verbatim : «Je suis touchée par notre marketing vers les prospects; c'est un peu comme si la marque me valorisait auprès de mes futurs clients » ; "notre communication externe est une vraie promesse ; je me sens responsable de porter ce message » « je suis fier de notre banque, sa communication nous valorise toujours; c'est bien dans un monde dans lequel le numérique est roi »). D’après nos observations, la stratégie marketing de l'enseigne avait donc un réel impact positif dans la valorisation des employés sans pour autant que ces derniers soient conscients d'en être 


\section{La relation client à l'heure du numérique : le cas du marketing bancaire}

la cible puisque leurs réponses démontraient toujours une perception de la stratégie marketing à destination de l'externe.

À la lecture des résultats, il est apparu que l'invitation du marketing de l'enseigne à engager et à développer la création d'un lien social n'était pas considérée comme une recommandation interne mais perçue comme une philosophie corporate à laquelle les employés adhéraient naturellement. En effet, les différents témoignages recueillis convergeaient : si la nature de la communication de la marque renforçait l'adhésion des employés à l'entreprise et leur loyauté, elle mettait également en exergue leur bien-être face à un environnement de travail n'offrant finalement aucune forme de dissonance cognitive. Ces résultats semblent donc démontrer qu'il existe une réelle source de création de valeur en matière d'échange social, à la fois entre les salariés et leur enseigne, et entre les salariés et leurs clients.

\subsection{La pondération d'épargnants, d'investisseurs et d'autres clients}

À ce stade, interroger des épargnants, des investisseurs et des clients avait pour objectif de compléter les résultats des entretiens internes avec un échantillon pondéré de catégories de clientèles parfois plus sensibles à la relation client, parfois plus indépendantes dans leur gestion, mais également capables de s'exprimer au sujet de la concurrence. Les clients de seuls pure players n'ont donc pas été considérés ici.

Les utilisateurs de services bancaires que nous avons interrogés sont habituellement fidèles à leur enseigne bancaire parce qu'ils sont avant tout fidèles à leur conseiller, un élément que renforce la complexité encore perçue de changer de banque, notamment lorsque des crédits sont en cours, et ce malgré la loi de mobilité bancaire (i.e. verbatim : «Ma conseillère est formidable»; «je n'ai pas le choix, j'ai tous mes crédits là-bas » ; "j'ai un très bon contact avec le personnel; je pourrais sans doute trouver mieux mais la situation me convient»). Ceci explique leur difficulté à finalement avoir une vision comparative fiable de l'offre globale (i.e. verbatim: "Je ne sais pas, je ne connais pas les autres banques»; « ils ont peut-être des taux plus attractifs; ou pas» ; " toute ma famille est là-bas, je suis le mouvement»), donc à se positionner dans une logique nomade face à la décision de changer de banque (i.e. verbatim : "Ils m'accompagnent surtout quand c'est compliqué»; "mon conseiller est très humain» ; "je ne sais pas »). Finalement, on constate surtout que la fidélité à l'enseigne est proportionnelle à sa légitimité technique (i.e. verbatim : «ils sont rassurants car factuels mais je n'y comprends rien "; "les chiffres vous savez, on leur fait dire ce que l'on veut; heureusement que j'ai confiance dans mon conseiller» ; «les calculs me dépassent mais ma conseillère, elle, me rassure car elle m'explique»; "je n'ai pas confiance dans les solutions numériques car on ne parle jamais avec la même personne»; «ces plateformes sont toutes déshumanisées, il faut toujours tout répéter, impossible d'avoir un suivi correct ») même si le concept de banque à proprement 
parler est devenu synonyme de défiance (i.e. verbatim : « une banque c'est un gangster légal » ; "un bâtiment sur lequel il est écrit 'banque'»; " une entreprise qui est présente quand tout va bien et absente quand tout va mal»). Ce qui ressort encore une fois, c'est que les rupteurs de loyauté comme les sources de fidélité à la marque sont souvent en lien avec l'action du conseiller (i.e. verbatim : "J'ai une bonne relation avec mon conseiller, autant la conserver» ; «je n'ai changé de banque que deux fois dans ma vie: la première fois parce que l'équipe dont j'avais l'habitude est partie et que sa remplaçante ne me comprenait pas, et la seconde fois parce que ma conseillère ne me répondait jamais»; « ma banque n'est pas parfaite et je pourrais sans doute trouver mieux ailleurs mais on se connait depuis longtemps, c'est agréable»; " un courtier fera certes mieux en termes de taux d'intérêt mais il ne sera pas là en cas de problème»; « ma seule crainte, c'est que mon conseiller qui me connaît si bien quitte mon agence»; «vous savez, un Livret $A$ c'est un taux identique pour tous, mais un conseiller c'est une relation unique»). Notons que les réponses fournies par les clients d'autres enseignes n'ont créé aucune interférence avec celles fournies par les clients de la marque étudiée. Ces données ont indiqué que si cet échantillon d'utilisateurs était fidèle à son enseigne bancaire principalement par absence de volonté de remettre en question sa loyauté à une banque, souvent à cause d'une vision indifférenciée de l'offre globale, le conseiller restait un levier essentiel à la fidélité des clients.

\section{Discussion}

Comme celle de ses concurrents, la communication bancaire de notre enseigne porte sur la valorisation d'un ensemble de produits intangibles et de services.

Mais elle a également pour objet l'image de cette valorisation dans la mesure où elle essaie d'influer sur la perception qu'a le public de la relation qu'il a ou pourrait avoir avec elle. La grande majorité des campagnes de notre marque a donc pour objectif d'amener le client à espérer des conditions d'écoute suffisantes pour débuter une discussion. Là où l'enseigne s'est singularisée en 2016, c'est lorsqu'elle a attribué à ses campagnes publicitaires locales un rôle prédominant dans la mobilisation de son propre personnel et de sa force de vente; car si les directeurs d'agences que nous avons interrogés considèrent que le seul moyen d'augmenter les parts de marché de façon significative est d'être directement au contact du prospect, ce que réfutent plusieurs managers d'un autre groupe interrogés à Niort le 19 juin 2017 au cours d'échanges informels qui, eux, parient sur la multiplication des outils numériques de gestion, ils ne sont en revanche pas conscients que les outils marketing nationaux sont élaborés en ce sens. Aussi, afin de recommander à la marque un ancrage identitaire qui vienne renforcer la création de valeur issue de l'échange social inhérent aux salariés de l'enseigne, nous proposons de recourir au prisme de Jean-Noël Kapferer (1988). 


\section{La relation client à l'heure du numérique :}

le cas du marketing bancaire

\section{Tableau 1 - Suggestion de prisme identitaire}

\begin{tabular}{|c|c|c|}
\hline RUBRIQUE & IDENTITE ACTUELLE & IDENTITE RECOMMANDEE \\
\hline $\begin{array}{l}\text { Physique } \\
\text { Attributs } \\
\text { tangibles }\end{array}$ & $\begin{array}{l}\text { - Personnification } \\
\text { réalisée au travers d'une } \\
\text { gamme de personnages } \\
\text { intergénérationnels. }\end{array}$ & $\begin{array}{l}\text { Le physique de la marque devrait } \\
\text { remettre le salarié au centre de la } \\
\text { relation sociale plutôt que de se fo- } \\
\text { caliser sur une représentation d'une } \\
\text { cible familiale rendue idéale par sa } \\
\text { composition et sa communication } \\
\text { intergénérationnelle. }\end{array}$ \\
\hline $\begin{array}{l}\text { Personnalité } \\
\text { Facette interne }\end{array}$ & - Banque familiale. & $\begin{array}{l}\text { L'enseigne devrait positionner } \\
\text { ses salariés comme «membres » } \\
\text { périphériques du noyau familial } \\
\text { (parrainages) tout en évitant toute } \\
\text { perception intrusive. }\end{array}$ \\
\hline $\begin{array}{l}\text { Relation } \\
\text { Face au client }\end{array}$ & $\begin{array}{l}\text { - Le précédent slogan } \\
\text { soulignait l'importance } \\
\text { de la relation sociale. } \\
\text { - Le nouveau slogan } \\
\text { responsabilise les clients } \\
\text { et invite à une gestion } \\
\text { multicanale. }\end{array}$ & $\begin{array}{l}\text { Le levier actuel de communication } \\
\text { (actionnariat) devrait basculer du } \\
\text { statut de proposition de vente unique } \\
\text { (USP) à celui d'avantage comparatif. } \\
\text { L'ancien levier (dialogue) devrait } \\
\text { être remis à l'honneur afin de valo- } \\
\text { riser la relation sociale face à l'offre } \\
\text { numérique. }\end{array}$ \\
\hline $\begin{array}{l}\text { Culture } \\
\text { Valeurs de } \\
\text { marque }\end{array}$ & $\begin{array}{l}\text { - Marque inter- et } \\
\text { multigénérationnelle. }\end{array}$ & $\begin{array}{l}\text { Cet axe doit pouvoir être conservé } \\
\text { mais en positionnant le conseiller } \\
\text { comme un lien rapprochant les } \\
\text { générations. }\end{array}$ \\
\hline $\begin{array}{l}\text { Reflet } \\
\text { Cible idéale }\end{array}$ & $\begin{array}{l}\text { - Multiple (générations) } \\
\text { mais intégrée (famille). }\end{array}$ & $\begin{array}{l}\text { La cible idéale devrait basculer de } \\
\text { l'intergénérationnel, donc familial, au } \\
\text { multigénérationnel, donc sociétal. }\end{array}$ \\
\hline $\begin{array}{l}\text { Mentalisation } \\
\text { Subjectivation }\end{array}$ & $\begin{array}{l}\text { - Assurance grâce à la } \\
\text { mutualisation. } \\
\text { - Transmission grâce à } \\
\text { l'inter-génération. }\end{array}$ & $\begin{array}{l}\text { La mutualisation peut fidéliser une } \\
\text { génération mais probablement pas } \\
\text { plusieurs générations. En revanche, } \\
\text { l'approche par la multi-génération } \\
\text { peut certainement à la fois rassurer } \\
\text { et fidéliser. }\end{array}$ \\
\hline
\end{tabular}

Cette suggestion identitaire propose de remettre le conseiller au cœur de l'échange social, en l'occurrence multigénérationnel. Pour cela, nous préconisons à l'enseigne d'engager les actions managériales suivantes :

1. La démarche consistant à parier sur la relation client créée par les employés pour lutter efficacement contre l'attractivité des pure players bancaires est source d'avantage concurrentiel durable. Cependant, il semble important de sensibiliser les directeurs d'agence à cette stratégie afin de s'assurer de 
l'absence de rupture entre une décision stratégique institutionnelle et une application locale.

Par exemple, la construction d'une relation humaine exige du temps, ce que l'on pourrait reprocher à des employés considérés comme trop diserts. Egalement, construire puis maintenir la relation bancaire autour des acteurs originels que sont le conseiller avec ses compétences et le client avec ses questionnements passe nécessairement par une responsabilisation du personnel comme acteur proactif de cette philosophie. Ce personnel doit être principalement tourné vers des profils non-investisseurs et privilégier le respect de sa cible par la pédagogie, la médiation et une relation personnalisée permettant de limiter la sensation d'intrusion dans la vie privée que révèle toute étude de comptes bancaires. À ce titre, l'évolution technologique doit rester ce qu'elle est, un moyen éphémère et renouvelable de gagner du temps et de faciliter certaines démarches, sans pour autant impacter dans une quelconque mesure la durabilité et la valeur intangible de la relation humaine. La rotation de personnel doit donc évidemment être limitée au maximum et l'enseigne doit rester vigilante quant à la nature de ses choix stratégiques de développement multicanal.

2. Intégrer et associer l'ensemble des acteurs bancaires au travers de formations internes de type "team building», donc proposées et organisées par les directions locales, afin que les conseillers continuent de développer leur attachement émotionnel à la marque, permettant ainsi de valoriser non plus l'outil de gestion mais bien sa représentation et son explication (Wallace et al., 2013 ; Ambler et Barrow, 1996; Balmer, 1995; Miles et Mangold, 2004 ; Gromark et Melin, 2011).

3. En interne toujours, s'assurer des possibilités locales d'évolution du personnel, donc sans forcément envisager la mutation, afin, justement, de conserver une capitalisation de la relation commerciale préalablement créée et permettant d'envisager la création de liens intergénérationnels.

4. Poursuivre la mise à jour technologique des outils de gestion mais en impliquant le personnel de façon à ce que ce dernier explique leur complémentarité et non leur substituabilité à l'action du conseiller.

5. Poursuivre la formation des personnels sur l'importance de la relation sociale, donc sur le risque d'influence des stéréotypes.

6. Mettre en place un outil de veille, notamment au travers de fréquentes enquêtes d'opinion auprès des parties prenantes de l'enseigne, afin d'identifier les sources possibles de dissonance cognitive entre la promesse d'un message publicitaire et la réalité de l'offre produits/services. Une telle veille permettrait également d'affiner la thématique des messages et d'optimiser leurs vecteurs de communication. Grâce à leur récurrence, ces observations offriraient des données empiriques et stochastiques fiables pour stabiliser et renforcer certains axes de communication, ainsi que pour détecter le plus tôt possible les signaux 


\section{La relation client à l'heure du numérique : le cas du marketing bancaire}

faibles annonciateurs de points d'inflexion ou l'apparition de modifications comportementales sociales, sociétales, voire organisationnelles (Grove, 1998).

\section{Conclusion}

À partir du terrain principal d'agences d'une enseigne bancaire et d'une série d'entretiens semi-directifs auprès de plusieurs acteurs et utilisateurs de services bancaires, entretiens tirés de la méthodologie appliquée à l'observation participante périphérique, cette recherche s'est attelée à vérifier l'hypothèse selon laquelle une telle marque serait capable de recréer du lien social entre ses conseillers et ses clients par une campagne marketing à destination officieuse de ses agents.

Nos résultats montrent que cette démarche est prometteuse, et ce à deux niveaux. Le premier niveau concerne les acteurs. Employés comme clients expriment un réel attachement à la relation humaine, les premiers par adhésion aux valeurs de leur enseigne, les seconds par besoin d'être rassurés. Quant au second niveau, il a permis de valider l'idée selon laquelle une banque pouvait mettre en œuvre une stratégie de communication marketing institutionnelle sans avoir recours à ses directions locales pour en assurer l'explication, la transmission et la mise en œuvre. Nonobstant ces résultats, il n'en demeure pas moins que cette dernière remarque soulève un point organisationnel important: isoler les directions régionales de telles stratégies, c'est prendre le risque de se priver d'un levier important et, surtout, de provoquer des dissonances entre ces mêmes directions et leurs subordonnés. Aussi, maintenant que nous avons pu valider l'impact positif d'une telle action, notre recommandation principale consiste ici à intégrer la totalité de la chaîne décisionnaire aux stratégies de communication de la marque afin de pouvoir bénéficier d'une adhésion de l'ensemble des employés aux valeurs humanistes de l'enseigne.

Les limites de cette étude résident essentiellement dans la nature et la taille de ses échantillons, ainsi que dans le choix d'une enseigne unique. Quand bien même notre terrain fut riche d'opinions dans leur diversité, une étude multi-marques et surtout longitudinale permettrait sans doute de mieux apprécier l'avenir du développement de la relation sociale face à la pression des technologies développées par les pure players bancaires. Une poursuite de cette recherche aurait donc du sens en termes d'identification des déclencheurs et des stabilisateurs d'une relation professionnelle, afin de renforcer les leviers marketing de la marque, peut-être par l'approche des «Big Five » (Modèle OCEAN, Sternberg, 1995). Par exemple, il serait intéressant de mesurer le pouvoir d'attraction d'un personnel en mutation vers d'autres enseignes sur la mobilité bancaire de ses clients. À ce titre, il serait sans doute pertinent de mieux segmenter nos échantillons, notamment sur la base du degré d'adoption des valeurs des marques de service de la part de leurs employés (Ind, 2007). Le profil démographique de nos répondants mériterait d'ailleurs certainement de venir compléter nos données. Egalement, il conviendrait sans doute d'explorer les influences de 
branding interne sur les campagnes associées (Liu et al., 2017), ainsi que sur les comportements des employés de type in-role / extra-role participant à la construction de la marque, le tout afin de mieux comprendre l'influence d'une orientation en marketing relationnel sur le capital marque (Yoganathan et al., 2015). Quant aux stratégies cross, omni et multi-canal, elles ne cesseront sans doute d'évoluer avec les technologies et l'intelligence artificielle, remettant continuellement en cause la solidité de la relation sociale entre un banquier et son client.

Quoi qu'il en soit, l'actualité semble pour le moment valider notre thèse puisqu'en octobre 2018, une campagne nationale de communication de cette marque bancaire mettait au cœur de sa stratégie marketing sa première place en matière de relation client, soit près de deux ans après la réalisation de notre étude...

\section{Bibliographie}

AMBLER T. et BARROW S. (1996), "The Employer Brand”, Journal of Brand Management, Vol. 4, $\mathrm{n}^{\circ}$ 3, p. 185-206.

BALMER J.M.T. (1995), “Corporate Branding and Connoisseurship”, Journal of General Management, Vol. 21, n 1, p. 24-46.

BELVAUX B. et NOTEBAERT J.-F. (2018), Cross canal et Omni canal, Dunod, Paris.

BOKE CHAKISO C. (2015), "The Effect of Relationship Marketing on Customers' Loyalty (Evidence from Zemen Bank)”, Emerging Markets Journal, Vol. 5, $\mathrm{n}^{\circ} 2$, p. 58-70.

CARLSSON HAUFF J., CARLANDER A., GAMBLE A., GÄRLING T. et HOLMEN M. (2014), "Storytelling as a Means to Increase Consumers' Processing of Financial Information", International Journal of Bank Marketing, Vol. 32, n 6, p. 494-514.

COLLIN-LACHAUD I. et VANHEEMS R. (2015), « Naviguer entre espace virtuel et réel pour faire ses achats : exploration de l'expérience de shopping hybride », Recherche et Applications en Marketing, Vol. 31, n², p. 43-61.

FORTEZZA F. et PENCARELLI T. (2011), “Experience Marketing : Specific Features and Trends. The Wish Days Case Study", Journal of Marketing Trends, Vol. 1, n 6, p. 57-69.

GARDES N., BEGUINET S. et LIQUET J.-C. (2013), « Du concept d'image à sa mesure, une approche par les représentations sociales », La Revue des Sciences de Gestion, Vol. 3, n²61/262, p. 157-166.

GATFAOUI S. (2007), «Comment développer une relation bancaire fondée sur la confiance? », Décisions Marketing, Vol. 46, p. 91-103.

GLEMAIN P. (2014), «Les proximités des microcrédits sociaux, une analyse en région Pays-de-la-Loire », Géographie, Économie, Société, Vol. 1, n 16, p. 33-46. 


\section{La relation client à l'heure du numérique :}

le cas du marketing bancaire

GROMARK J. et MELIN F. (2011), "The Underlying Dimensions of Brand Orientation and its Impact on Financial Performance", Journal of Brand Management, Vol. 18, p. 394-410.

GROVE A. (1998), Only the Paranoid Survive, London, Profile Books Ltd.

HEINONEN K. (2014), "Multiple Perspectives on Customer Relationships", International Journal of Bank Marketing, Vol. 32, n 6, p. 450-456.

HLADY-RISPAL M. (2015), «L'étude de cas : une stratégie de recherche en gestion », Revue Française de Gestion, Vol. 8, n² 253, p. 251-266.

HOMANS G.C. (1958), "Social Behavior as Exchange”, American Journal of Sociology, Vol. 63, p. 597-606.

IND N. (2007), Living the Brand: How to Transform Every Member of Your Organization into a Brand Champion ( $3^{\text {rd }}$ Edition), London, Kogan Page.

JULIEN A. (2013), «La valeur apportée par un réseau social Brick and Mortar: Les points passerelle du Crédit Agricole », Gérer \& Comprendre, $\mathrm{n}^{\circ} 113$, p. 40-50.

KAPFERER J.-N. (1988), « Maîtriser l'image de l'entreprise : le prisme d'identité », Revue Française de Gestion, Vol. 71, p. 76-82.

KNOX S. et FREEMAN C. (2010), "Measuring and Managing Employer Brand Image in the Service Industry", Journal of Marketing Management, Vol. 22, $\mathrm{n}^{\circ} 7 / 8$, p. 695-716.

LIU G., KO W.W. et CHAPLEO C. (2017), "Managing Employee Attention and Internal Branding”, Journal of Business Research, Vol. 79, p. 1-11.

LÖHNDORF B. et DIAMANTOPOULOS A. (2014), "Internal Branding : Social Identity and Social Exchange Perspectives on Turning Employees into Brand Champions", Journal of Service Research, Vol. 17, n 3, p. 310-325.

MERCANTI-GUERIN M. (2011), «Crise du secteur bancaire et portrait de la banque idéale », La Revue des Sciences de Gestion, Vol. 3, n²49/250, p. 57-64.

MILES S.J. et MANGOLD G. (2004), "A Conceptualization of the Employee Branding Process", Journal of Relationship Marketing, Vol. 3, n² 2/3, p. 65-87.

N'GOALA G. (2010), «À la découverte du côté sombre des relations de service... ou pourquoi les relations durables et exclusives s'autodétruisent », Recherche et Applications en Marketing, Vol. 25, $\mathrm{n}^{\circ}$ 1, p. 3-31.

NOTEBAERT J.-F. et ATTUEL-MENDES F. (2010), « La résistance du client au travail sur Internet : le cas bancaire français », Management \& Avenir, Vol. 31, p. 209-227.

NYMAN H. (2014), "The Added Value of Service Provision”, International Journal of Bank Marketing, Vol. 32, n 6, p. 457-476. 
PAPASOLOMOU I. et VRONTIS D. (2006), "Building Corporate Branding through Internal Marketing: The Case of the UK Retail Bank Industry", Journal of Product and Brand Management, Vol. 15, $\mathrm{n}^{\circ}$ 1, p. 37-47.

RENAULT S. (2017), "Crowdsourcing, la foule en question(s) », Gérer \& Comprendre, $\mathrm{n}^{\circ} 129$, p. 45-57.

RICO J.-C. et SUEUR I. (2014), « La fidélité des clients professionnels envers leur banque: déterminants et implications managériales », Revue Française du Marketing, Vol. 249, p. 7-22.

SALERNO A. (2001), « Une étude empirique des relations entre personnalisation, proximité dyadique et identité de clientèle », Recherche et Applications en Marketing, Vol. 16, $\mathrm{n}^{\circ}$ 4, p. 25-46.

SALERNO A. (2002), Le rôle de la congruence des valeurs marque-consommateur et des identifications sociales de clientèle dans l'identification de la marque, Proceedings of The Conference of The French Association of Marketing, p. 399-427.

SOULE B. (2007), « Observation participante ou participation observante? Usages et justifications de la notion de participation observante en sciences sociales », Recherches Qualitatives, Vol. 27, $\mathrm{n}^{\circ}$ 1, p. 127-140.

STERNBERG R.J. (1995), In Search of the Human Mind, Florida, Harcourt Brace \& Company.

STRAUSS J.-L. (2013), «Qu'est-ce que l'innovation dans la banque ?», Revue Banque, Vol. 762.

TOURNOIS N. (2017), Le Marketing bancaire face aux nouvelles technologies, Elsevier Masson, Gestion Pratique Entreprise, Paris.

VALETTE-FLORENCE R. et DE BARNIER V. (2013), "Towards a Micro Conception of Brand Personality: An Application for Print Media Brands in a French Context", Journal of Business Research, Vol. 66, n 7, p. 897-903.

VANHEEMS R. (2009), « Distribution multicanal : Pourquoi les clients mixtes doivent faire l'objet d'une attention particulière », Décisions Marketing, Vol. $55, \mathrm{n}^{\circ} 41$, p. $41-52$.

VANHEEMS R. (2013), "La distribution à l'heure du multicanal : Une redéfinition du rôle du vendeur », Décisions Marketing, Vol. 69, n43, p. 43-59.

WALLACE E., BUIL I. et CHERNATONY L. (2013), "Brand Orientation and Brand Values in Retail Banking”, Journal of Marketing Management, Vol. 29, $\mathrm{n}^{\circ}$ 9/10, p. 1007-1029.

YOGANATHAN D., JEBARAJAKIRTHY C. et THAICHON P. (2015), "The Influence of relationship Marketing Orientation on Brand Equity in Banks", Journal of Retailing and Consumer Services, Vol. 26, p. 14-22. 